\title{
La canalisation de la Moselle, une œuvre résultant de la collaboration pacifique des pays européens
}

\author{
The canalization of the Moselle \\ as an example of peaceful collaboration \\ between European countries
}

IAI BHNO KRETZSSCHMAR,

ESCHWEILER/AACHEN

\begin{abstract}
L'article décrit l'ensemble des aménagements opérés et des oumrages construits on $\dot{a}$ construire sar la Moselle en vue de canaliser Ia rivière, d'améliorer ainsi la navigation sur cet affluent du Rhin, et de produire de l'énergie électrique en utilisant la dizaine d'étages de retenue sifnée sitr les territoires allemand et luxembourgeois. I'étage de Trèves est décrit plus complètement (barrage, écluses, centrale) et mention est faite de l'adaptation des ouvrages au paysage. Deux paragraphes sont réservés au fonctionnement des centrales par éclusées et aux projets de centrales d'accumulation par pompage.
\end{abstract}

\begin{abstract}
This article describes the various projects and both present and future works connected with the canalization and the improvement of navigation conditions in the Moselle, an importunt tributary of the Rhine. Electric power projects using the ten storage stages on German and Luxembourg territory are also reviewed. A fuller description is given of the stuge at Trèves, including the barrage, locks and power station, and attention is draun to the care taken to blend the structures in wilh the local landscape. Two paragraphs are given over to a discussion of power station operation by releases of water from locks, and pumped storagc power plant projects.
\end{abstract}

\section{INTRODUCTION}

La Moselle, un affluent du Rhin, a une longueur totale de $514 \mathrm{~km}$. Sa source se trouve dans la partie la plus méridionale des Vosges, entre le Ballon d'Alsace et le Drumont. Près de Remiremont, la Moselotte se jette dans la Moselle et c'est à partir de là également qu'elle forme, sur une longueur de $34 \mathrm{~km}$ environ, la frontière entre le Grand-Duché de Luxcmbourg et l'Allemagne de l'Ouest. Elle rejoint le Rhin près de Coblence. Ses affluents les plus importants sont la Meurthe et la Saar à droite, ainsi que l'Orne, la Sure, la Kyll, la Lieser et l'Alf à gauche.

Nous trouvons les plus jolis sites entre Trèves et Coblence où, par un cours sinueux, la Moselle a creusé son lit dans le massif schisteux rhénan, à travers des vallées profondes. C'est également la région principale de la cultude de la vigne, crée par les Romains.

A partir de Frouard, la Moselle est navigable pour des petits bateaux sur une longueur de 
$344 \mathrm{~km}$. Elle est reliée par des canaux à la Saar, àu Rhin, à la Meuse, à la Marne ainsi qu’à la Saône. Autrefois, des petits bateaux assuraient le transport des voyageurs entre Trèves et Coblence. Le canal de la Moselle a été construit en amont de Metz [1, 2].

\section{1. - La Moselle comme voie de communication entre les régions industrielles européennes.}

Le 27 octobre 1956, la France, le Luxembourg et l'Allemagne de l'Ouest, ont signé une conven-

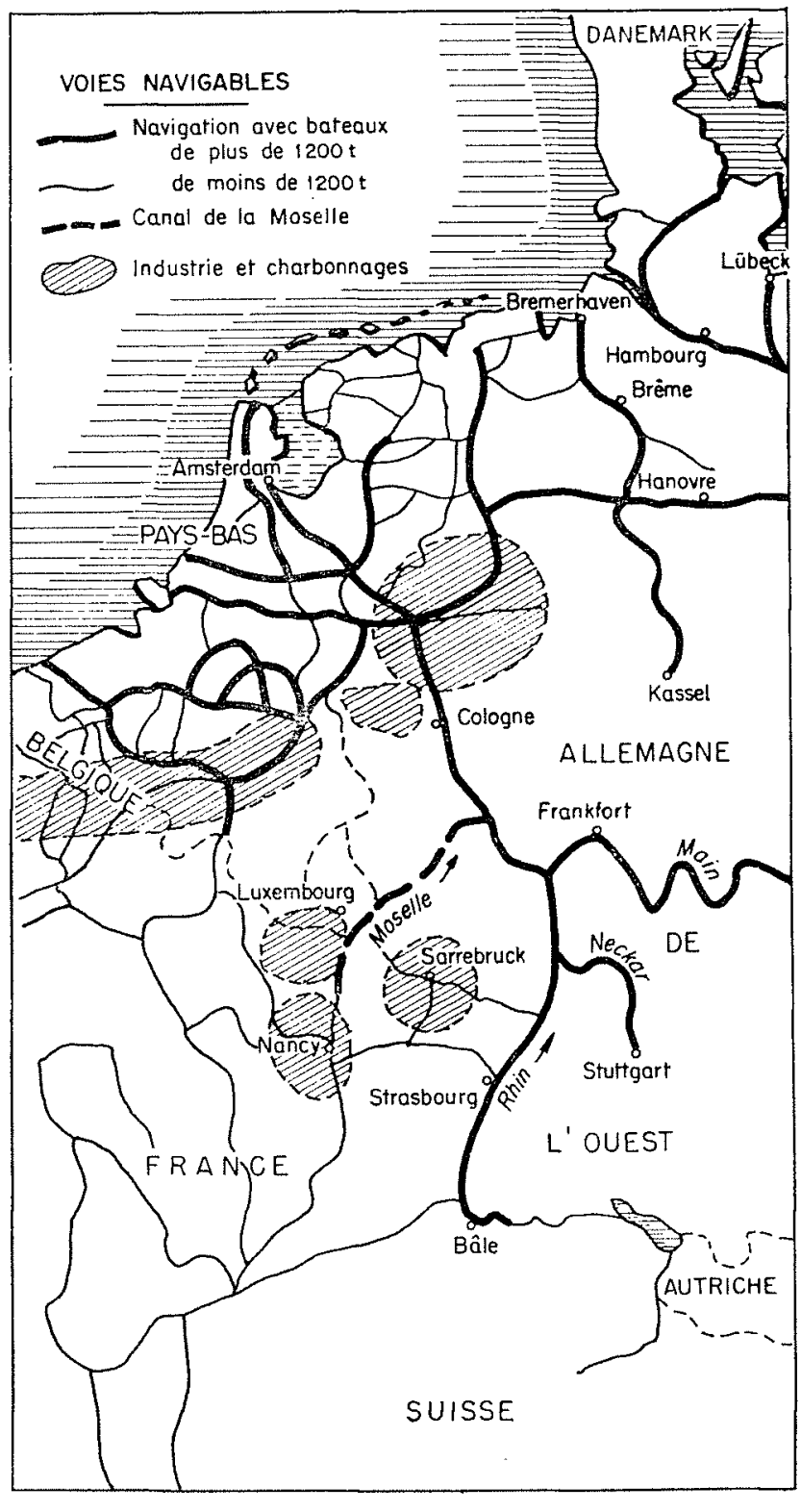

Fic. 1

La voie fuviale de Ia Moselle, lien entre les centres industriels et charbonniers d'Europe occidentale. lion sur le développement, la canalisation et l'aménagement des centrales hydrauliques pour la production d'énergie électrique dans la section située entre Thionville et Coblence.

Cette nouvelle voie de navigation, créée pour des bateaux jusqu'à $1500 \mathrm{t}$, reliera étroitement la région industrielle lorraine au Rhin, au bassin de la Ruhr ainsi (fu'aux ports hollandais et belges. Ia figure 1 donne un apercu de cette imporlante voie de communication fluviale. On y voit retracés les parcours navigables pour des bateaux de plus de $600 \mathrm{t}$ ainsi que ceux accessibles aux bateaux de moins de $600 \mathrm{t}$. La section de la Moselle située entre Thionville et Coblence a une longueur totale d'environ $270 \mathrm{~km}$. La dénivellation moyenne sur ce troncon est de $50 \mathrm{~m}$. Après achivement des travaux de canalisation, il scra navigable pour des bateaux de $1500 \mathrm{t}$.

Il est difficile de donner une estimation du trafic fluvial futur sur ce parcours. D'après les experts francais, le trafic annuel escomptable sera de 11 millions de tonnes environ. L'expérience a montré que de telles valeurs sont souvent inf'́rieures aux valeurs effectivenent atteintes à la suite de la mise en service d'une voie fluviale aussi importante.

Il faut remarquer que dans le cadre d'une collaboration pacifique des pays européens, ce projet est maintenant en voie de réalisation. Les nombreux plans de construclion et les programmes à long terme qui ont été établis par différents pays d'Europe après 1945 démontrent nettement la volonté de reconstruire ce continent tant éprouvé par la deuxième guerre mondiale. L'accélération de la production charbonnic̀re représentait le point le plus important de ces travaux de planification, mais ceci a conduit en France, en Belgique et en Allemagne, à des dépôts de charbon qui ne trouvent pas d'acquéreur actuellement. Une grande importance a cté accordée à l'augmentation de l'alimentation de l'Europe en énersie, dans ces lravaux dils d'apres-guerre. Dans ce domaine, c'est surtout lo plan commun des pays européens qui tend à équilibrer l'énergie électrique entre la France, les pays du Benelux, les nations alpines ainsi que l'Allemagne $[3,13]$ et qui a dé développé avec succes depuis 1945

Des succes remarquables ont été oblenus dans le cadre du plan commun des pays de l'Europe occidentale. Durant les annces apres 1945, le manque d'énergic électrique dans les pays d'Europe a conduit a des mesures de restriction paralysant à leur tour le développement de l'industrie européenne $[5,6,9,11,12]$.

Les chifres suivants démontrent les résultats obtenus depuis lors : en septembre 1958, ont été connectés en parallèle pour la pointe matinale, vers 11 heures, la France, le Benelux, la Suisse, 
l'Autriche, l'Italie et l'Allemagne. La puissance des centrales hydrauliques et thermiques européennes travaillant en connexion était de plus de 30 millions de $\mathrm{kW}$. La puissance des zones des U.S.A. travaillant en connexion, atteignant à cette époque environ $25.10^{6} \mathrm{~kW}$ en pointe, fut donc ainsi dépassée.

La production officielle de l'Electricité de France était en 1923 de $7490.10^{6} \mathrm{kWh}$, dont cnviron $55 \%$ par des usines thermiques. Elle ćtait de $19700.10^{6} \mathrm{kWh}$ en 1939 (dont environ $42,5 \%$ par usines thermiques [14], alors qu'en 1955, la production de l'Electricité de France s'élevait à presque $50.10^{9} \mathrm{kWh}$ et l'on estime que pour $1965 / 66$, les besoins en énergie électrique de la France atteindront $100.10^{\circ} \mathrm{kWh}[15]$.

La production d'énergie électrique des pays travaillant dans le cadre de l'union des pays de l'Europe occidentale s'élevait en 1948 à environ $58194 \mathrm{GWh}(52 \%)$ dans les centrales hydrauliques et à 53781 GWh dans les centrales thermiques, soit, au total, à presque $111 \mathrm{TWh}$ $\left(1 \mathrm{GWh}=10^{9} \mathrm{Wh}, 1 \mathrm{TWh}=10^{12} \mathrm{Wh}\right)$. En 1957 , clle dépassait 218 TWh $[16]$.

Afin de permettre une comparaison, nous pouvons dire que la production des U.S.A. durant l'année 1925 s'élevait à environ $61 \mathrm{TWh}$, dont $64 \%$ dans des usines thermiques. Dix années plus tard, les besoins s'élevaient à $95 \mathrm{TWh}$, dont $60 \%$ fournis par des usines thermiques. Dans les Etats-Unis, l'augmentation annuelle durant l'époque de 1925 à 1935 était de 3,4 TWh. En 1945, la consommation s'élevait à $220 \mathrm{TWh}$ et en 1957 le bilan était de 631 TWh $(80 \%$ par des usines thermiques). D'après les estimations, les besoins des Etats-Unis en électricité pour l'année 1975 s'élèveraient à 2400 TWh, 85,5 \% étant fournis par des usines thermiques [18].

En vertu du fait bien connu que les besoins en ćlectricité des pays industriels d'Europe doublent en sept à dix années, on voit qu'il est nécessaire de développer l'exploitation de l'électricité en Europe. Dans ce cadre, la signification de la canalisation de la Moselle, ainsi que des centrales à aménager, devient également plus claire.

\section{2. - Débits utilisables de la Móselle.}

En 1958, 57535 GWh onl été produits par les centrales électriques publiques de l'Allemagne de l'Ouest, avec une production de 19,7 \% seulement fournie par les installations hydrauliques. Les centrales industrielles privées ont produit dans la même année 36 \$76 GWh $4 \%$ par les installations hydrauliques). Les installations des chemins de fer de l'Allemagne Fédérale onl fourni $1059 \mathrm{GWh}$, dont 37 \% par des installations hydrauliques.

En considérant tous les producteurs d'èlectricité de l'Allemagne de l'Ouest, nous obtenons pour 1958 la production suivante :

Production des centrales

thermiques ..... $82108 \mathrm{GWh}=86,2 \%$

Production des centrales

hydrauliques .... $13162 \mathrm{GWh}=13,8 \%$

Production tolale de

l'Allemagne de l'Ouest $95270 \mathrm{GWh}=100 \%$

dont :

Production par les entreprises de distribution d'Electricité (EVU).......

Production par les centrales d'industries (IVA) ................

Production par les chemins de fer fédéraux ............... $60,4 \%$ $38,5 \%$

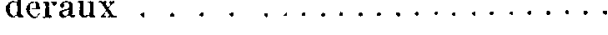

La participation de l'hydraulique à la producfion d'énergie électrique allemande est, suivant les débits disponibles, de 18 à $20 \%$. Pendant ces dernières années, l'énergie hydraulique s'est limitée à ce pourcentage. De 1936 à 1954, la production totale a subi une augmentation de $181 \%$, alors que celle des centrales hydrauliques n'était que de $70 \%$ [19]. Cette infériorité dans la production d'énergie hydraulique est due principalement à la situation particulière des années d'après-guerre et aux délais de construction plus longs des installations hydrauliques. Afin de remédier aux conséquences désastreuses de la guerre pour la production d'énergie électrique de l'Allemagne de l'Ouest, il fallait avant tout reconstruire les centrales thermiques détruites. Etant donné que, pendant les années de guerre, aucune installation nouvelle n'avait été construite et qu'en majeure partie, les installations à moyenne pression dominaient, la deuxième phase du programme concernant les centrales thermiques fut marquée par la réalisation d'importantes extensions. Ici, on peul citei comme exemple la centrale thermique (au lignite), de Goldenberg-Werk, près de Cologne. Cette installation, construite en 1913, était, lors de la déclaration de guerre, la plus grande du monde fonctionmant au lignite, avec ses $18 \mathrm{grou}-$ pes de turbines à moyenne pression $\left(15 \mathrm{~kg} / \mathrm{cm}^{2}\right.$, température de la vapeur $350^{\circ} \mathrm{C}$ ) ayant une puissance totale de $533 \mathrm{MW}$. La production de vapeur s'effectuait dans 100 chaudières verticales à grilles de foyer en marches d'escalier el tirage naturel. La production de chaque chaudicre chait de $30 \mathrm{t} / \mathrm{h}$. La centrale de Goldenberg avait 12 che- 
minées d'une hauteur de $125 \mathrm{~m}$. Plusieurs bombardements, notamment le raid anglo-américain d'octobre 1944, ont paralysé complètement cette centrale. Plus de 2000 bombes, - y compris celles qui n'ont pas éclaté, — ont été dénombrées sur l'emplacement de la centrale ainsi que sur les usines de briquettes et les mines de lignite situées alentour. Les travaux de reconstruction ont duré jusqu'en 1948. Après une durée de reconstruction, remarquablement courte, de ringt-deux mois, cette installation à moyenne pression fit l'objet, en 1950, d'une extension complémentaire a haute pression. Cette extension prévue pour une pression de $125 \mathrm{~kg} / \mathrm{cm}^{2}$ et $500^{\circ} \mathrm{C}$, comprend trois groupes de centrales munis chacun de quatre chaudières au poussier de charbon, de deux turbines auxiliaires (de $35 \mathrm{MW}$ chacune) et d'une turbine à pré-chauffage. La puissance auxiliaire totale est de $225 \mathrm{MW}$. La production unitaire des chaudières au poussier de charbon est de $160 / 175 \mathrm{t} / \mathrm{h}$. Après achèvement de ces travaux, la centrale de Goldenberg fut en 1952 agrandie par deux unités de $100 \mathrm{MW}$ (à $125 \mathrm{~kg}$ / $\mathrm{cm}^{2}$ et $530^{\circ} \mathrm{C}$ ). Ces chaudières (deux chaudières a circulation naturelle munies chacune de huit broyeurs à poussier) fournissent $365 / 400 \mathrm{t} / \mathrm{h}$ de vapeur. Ces quelques indications sont un exemple de la tendance dans le développement de la construction de centrales en Allemagne de l'Ouest après la deuxième guerre mondiale. Ce dévelop- pement tendait surtout vers l'agrandissement de centrales thermiques, l'Allemagne de l'Ouest ćtant très riche en matières combustibles solides, soit 68 milliards de tonnes de houille $(20 \%$ des gisements européens et 63 milliards de tonnes de lignite $(42,2 \%)$.

Les statistiques [19] démontrent que $53 \%$ environ des aménagements hydrauliques de l'Allemagne de l'Ouest, avec une capacité de production de $1950 \mathrm{MW}$, se trouvent seulement à l'état d'avant-projets. Des centrales d'énergie de pointe peuvent être amćnagées jusqu'à 64 \% environ avec une puissance de plus de $2000 \mathrm{NW}$.

La comparaison avec la richesse en énergie hydraulique de la France dans les Pyrénées, les Alpes, le Massif Central, montre que l'Allemagne est relativement pauvre en énergie hydraulique. La majeure partie de celle-ci se trouve concentrée dans le sud de l'Allemagne (Bavière).

L'interconnexion des centrales thermiques et hydroélectriques a permis l'aménagement d'usines hydrauliques à débits turbinés plus élevés, de façon à utiliser au maximum l'énergie nalurelle disponible. Le « fonctionnenent par éclusées » dans des centrales en cascade a été adopté de plus en plus au cours de ces dernières annócs - comme, par exemple, sur l'Isar, la Lech et le Neckar -; il permet la combinaison centrales au fil de l'eau - centrales sous retenue, parliculièrement mise en valeur par l'interconnexion

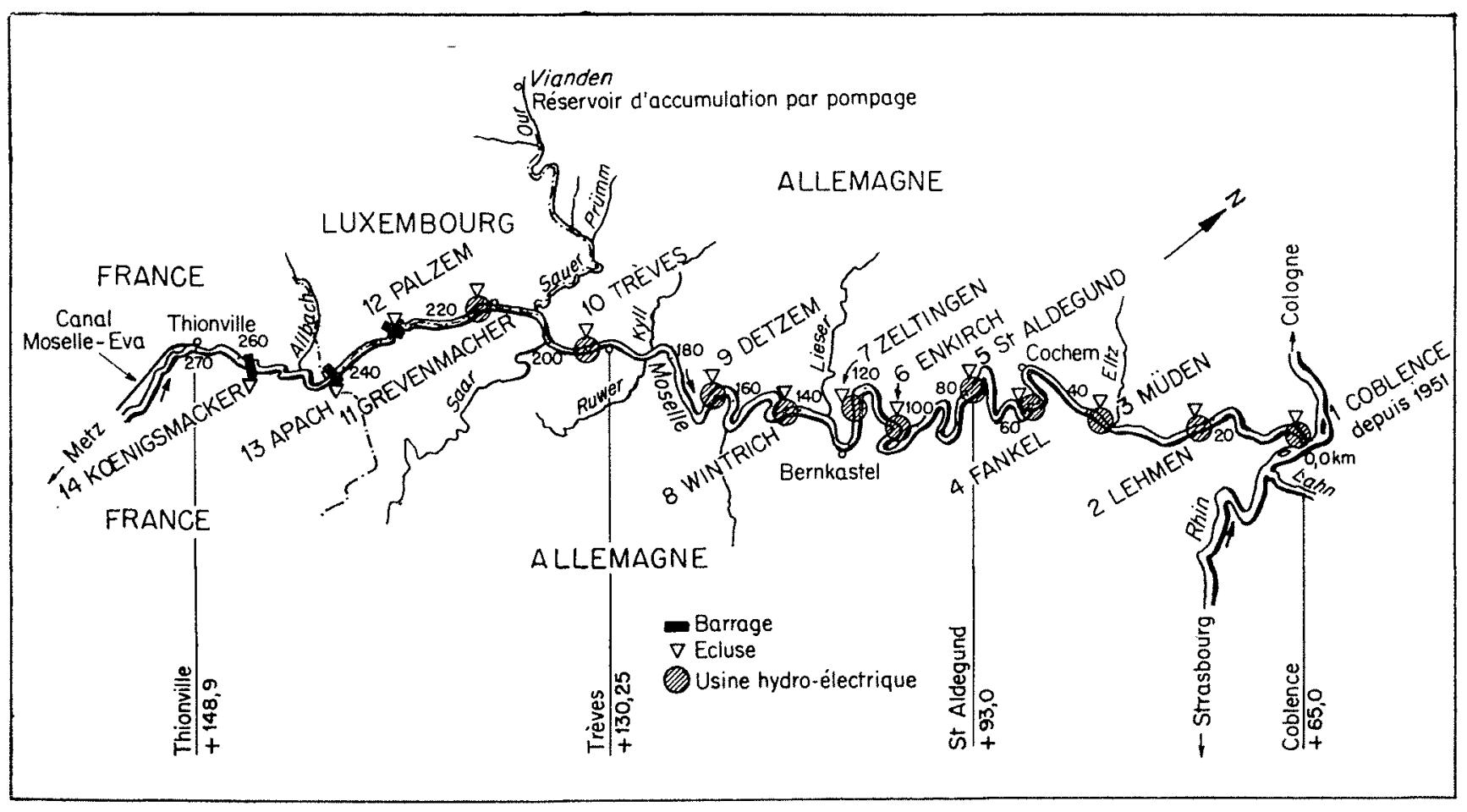

Fil. 2

Canalisation de la Moselle entre Thionville el coblence avec aménagement de 13 étages de retenue et de 10 centrales hydrauliques. 


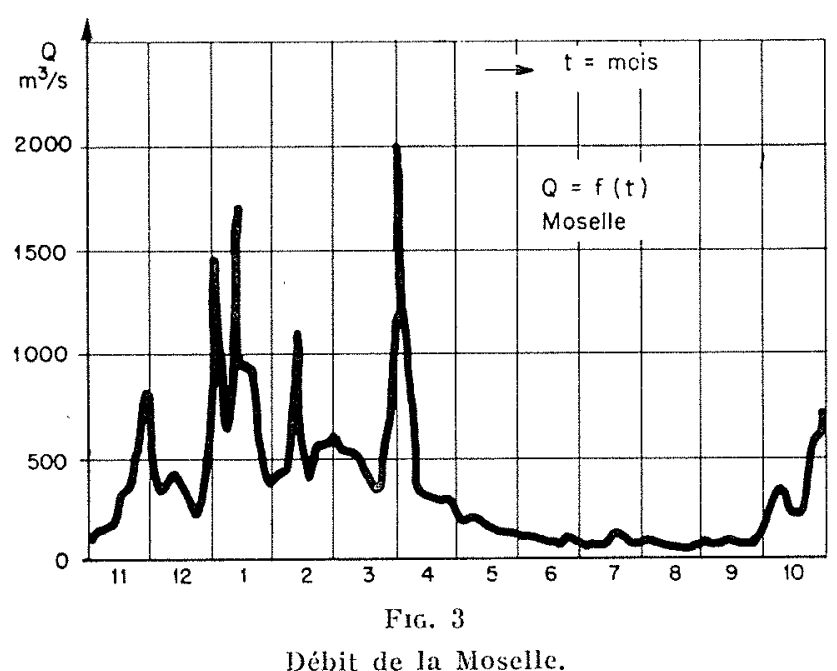

Limnimetre de Cochem (bassin versant de $27100 \mathrm{~km}^{2}$ ) : $\mathrm{Q}_{\mathrm{max}}=4100 \mathrm{~m}^{3} / \mathrm{s} ; \mathrm{Q}_{\mathrm{min}}=25 \mathrm{~m}^{3} / \mathrm{s}$; debit d'hiver $2 / 3$.

avec les centrales d'accumulation par pompage et il présente des avantages économiques.

La pointe hivernale de puissance, par rapport au régime nocturne, est en Allemagne de l'Ouest de 50 à $250 \%$. Par rapport à la pointe de nuil augmentée de l'accumulation par pompage, la pointe de puissance diurne est encore supérieure de $33 \%$ [19]. Pour satisfaire aux consommations de pointe, on a besoin de peu de $\mathrm{kWh}$, mais de beaucoup de kW. Ici, ce sont les centrales à retenue qui conviennent le mieux [19]. En Allemagne de l'Ouest - y compris les usines sur l'Ill (Vorarlberg) - on ne dispose que de $5 \%$ environ de la production annuelle en kWh pour l'énergie de pointe; la construction de centrales de retenue ainsi que de centrales à accumulation par pompage y est donc de première nécessité.

D'autre part, l'aménagement des centrales au fil de l'eau se poursuit aussi de façon intensive en Allemagne. Pour ces installations, les problèmes à résoudre se situent souvent dans le domaine de la régularisation des crues et des rivières, de l'alimentation en eau potable ainsi que de l'amćnagement pour la navigation. Pour ces aménagements à buts multiples, l'installation supplémentaire de centrales est d'une économie bien comprise.

Pour la canalisation de la Moselle, les chutes suivantes sont prévues :

France :

2 chutes (Konigsmacker, Apach).

Luxembourg :

2 chutes (Palzem, Gravenmacher).

Allemagne :

9) chutes (Trier, Detzem, Wintrich, Zeltingen, Enkirch, St. Aldegund, Fankel, Müden, I.ehmen).
Parmi les 13 aménagements, 10 (9 en Allemagne et 1 au Luxembourg) seront équipés de centrales hydroélectriques qui sont indiquées sur la figure 2. La centrale de Coblence (4 turbines Kaplan verticales, d'une puissance totale de $16 \mathrm{MW}$ ) est en service déjà depuis 1951 .

La Moselle étant une rivière de montagne d'altitude moyenne, ses débits disponibles varient dans les limites assez grandes. La figure 3 montre les conditions hydrologiques pour un débit annuel moyen. Le débit maximal à l'étiage est de $25 \mathrm{~m}^{3} / \mathrm{s}$ environ; le débit de crue maximal est de $4100 \mathrm{~m}^{3} / \mathrm{s}$ environ. Le débit moyen est de $300 \mathrm{~m}^{3} / \mathrm{s}$, ce qui donne un volume annuel de $9.10^{9} \mathrm{~m}^{3}$ environ. Il est essentiel que les deux tiers environ du débit s'écoulent en hiver, c'està-dire pendant la période où la charge dans le réseau commun est très élevée. La période des basses eaux se situe surtout pendant les mois d'été et d'automne.

Les variations du plan d'eau étant très rapides, la Moselle peut être considérée comme présentant des dangers de crues. Il a été tenu compte de ce fait pour la construction des barrages. En aval de l'embouchure de la Saar, c'està-dire dans le tronçon situé entre Trèves et Coblence, les affluents de la Moselle ne sont plus qu'insignifiants. Sur ce tronçon, d'une longueur de $200 \mathrm{~km}$, les débits varient peu.

\section{3. - Dispositions générales des aménage- gements.}

Sur la partie à canaliser de la Moselle, située entre Thionville et Coblence et longue de $270 \mathrm{~km}$ environ, la dénivellation moyenne, y compris l'aménagement de Coblence qui fonctionne déjà, est de $90 \mathrm{~m}$ environ. Chaque aménargement comprendra une grande écluse de navigation et à côté une petite ćcluse $p$ ur les péniches. Des garages suffisamment vastes, en amont et en aval, assurent un dégagement rapide de la navigation. La construction d'une deuxic̀me écluse sera prévue plus tard pour chacun des aménagements.

Les barrages situés sur le territoire allemand comprendront trois pertuis et une largeur totale utile de $120 \mathrm{~m}$. La centrale sera implantée à côté du barrage.

Les divers aménagements situćs entre 'Thionville et Coblence ne se prêtent pas tous à l'établissement de centrales électriques. Du côté français, le débit de la Moselle est nettement inférieur à celui sur les territoires luxembourgeois et allemand, où le débit est presque doublé par les eaux venues de l'ouest de l'Eilel par la rivière la Sure et ses affluents le Prïn el l'Our, ainsi que par la Sarre venanl du sud. Comme le montre la figure 2, les possibilités de chute pour 
les aménagements à l'aval de Trèves sont également meilleures qu'en amont. Des centrales seront donc implantées surtout dans la région de Trèves-Coblence. Les conditions d'écoulement itant les mêmes sur tout le tronçon, long de $200 \mathrm{~km}$ environ, toutes les centrales seront prévues avec un même débit turbinable $\left(380 \mathrm{~m}^{3} / \mathrm{s}\right)$. Pour une ouverture plus grande des turbines, ce

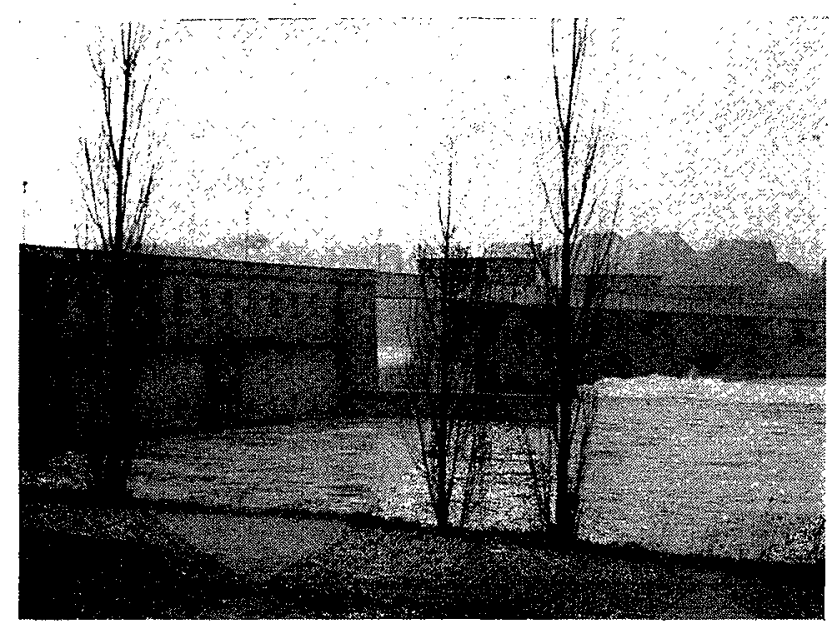

a) Vue d'aval.

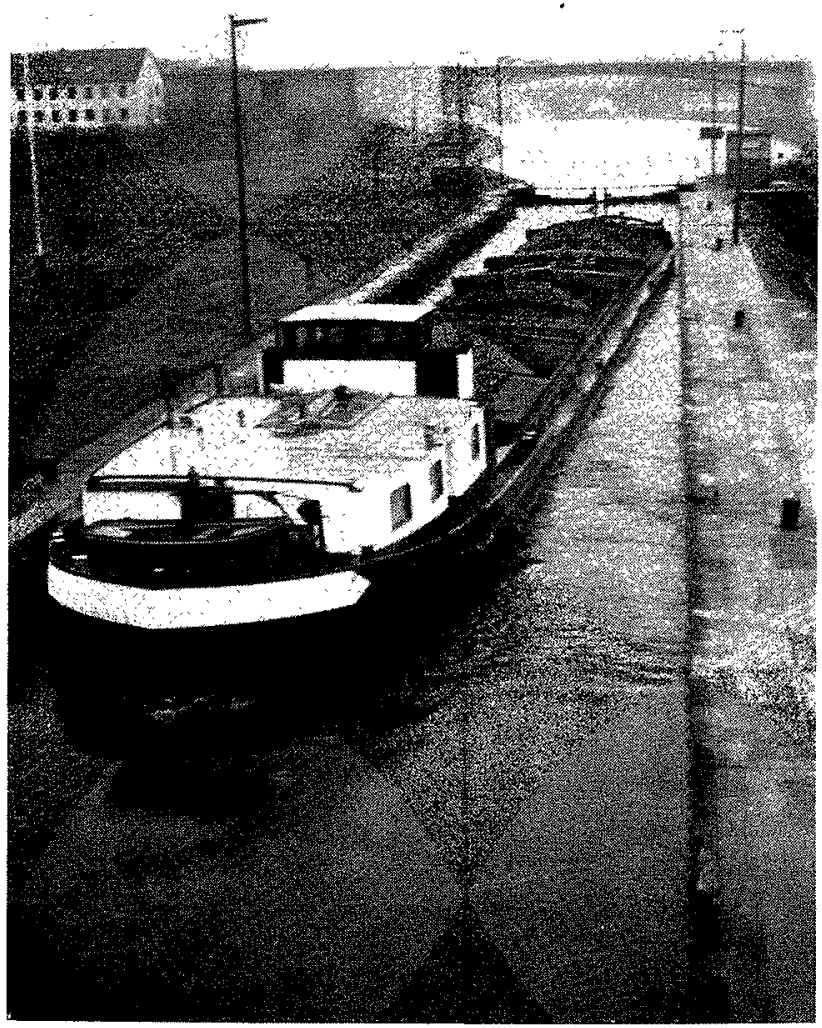

débit s'élève à $420 \mathrm{~m}^{3} / \mathrm{s}$. La dénivellation totale du tronçon indiqué plus haut est de $70 \mathrm{~m}$ et elle est répartie sur 10 chutes, y compris l'aménagement de Coblence fonctionnant déjà. On dispose donc d'une puissance totale de $165 \mathrm{MW}$, avec une production annuelle d'environ $750.10^{6} \mathrm{kWh}$. Le débit turbinable est disponible pendant 90 jours sur une période de plusieurs années.

Comme il a déjà été dit, on peut compler sur les deux tiers de l'énergie électrique de la Moselle pendant les six mois d'hiver. Ceci est très avantageux pour l'interconnexion avec les forces hydrauliques du sud de l'Allemagne, qui présentent leur maximum pendant les mois d'été. Mais il est désavantageux qu’à partir d'un débit

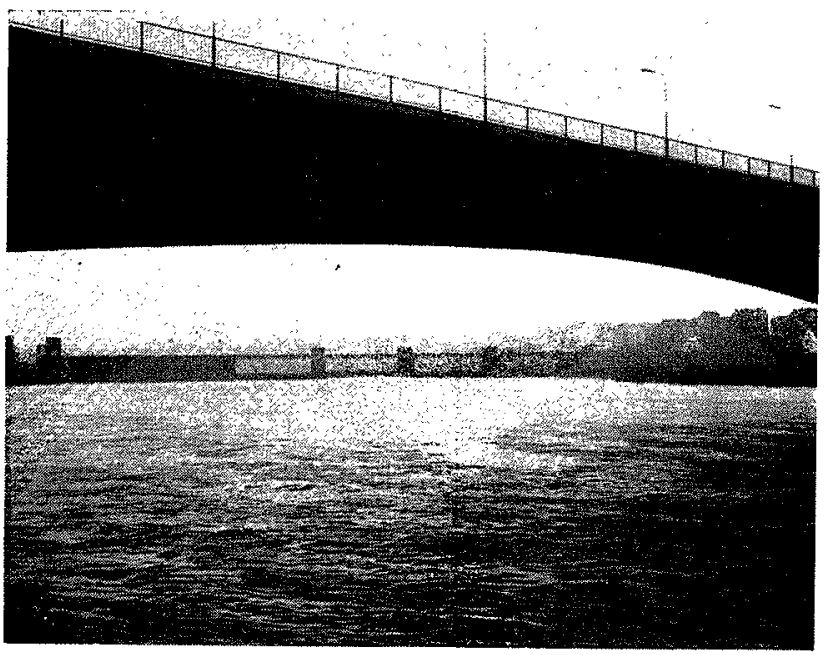

c) Vue d'amont.

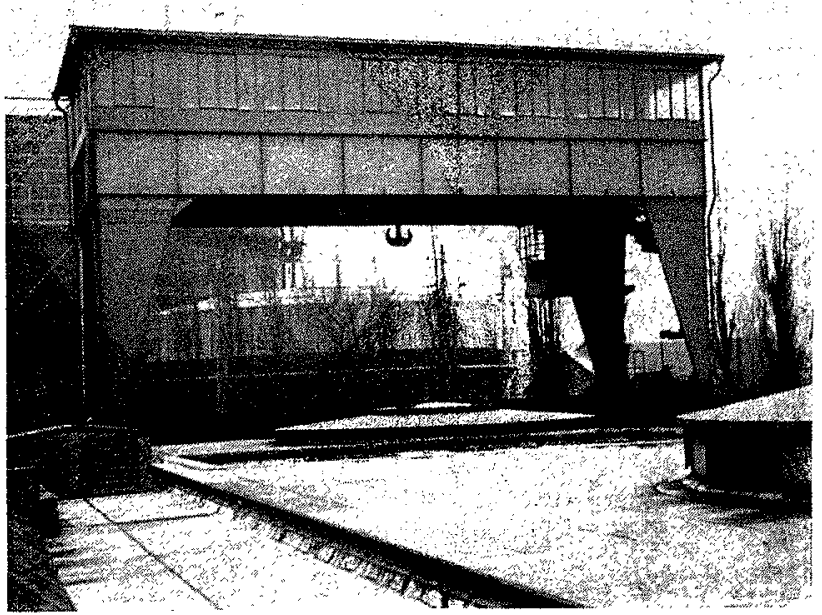

d) Porique de levage.

Fig. 4

Aménagements de Coblence.

Débit $=380 \mathrm{~m}^{3} / \mathrm{s} ;$ chute $=4,2 \mathrm{~m} ;$ puissance $=16 \mathrm{MW}$. 


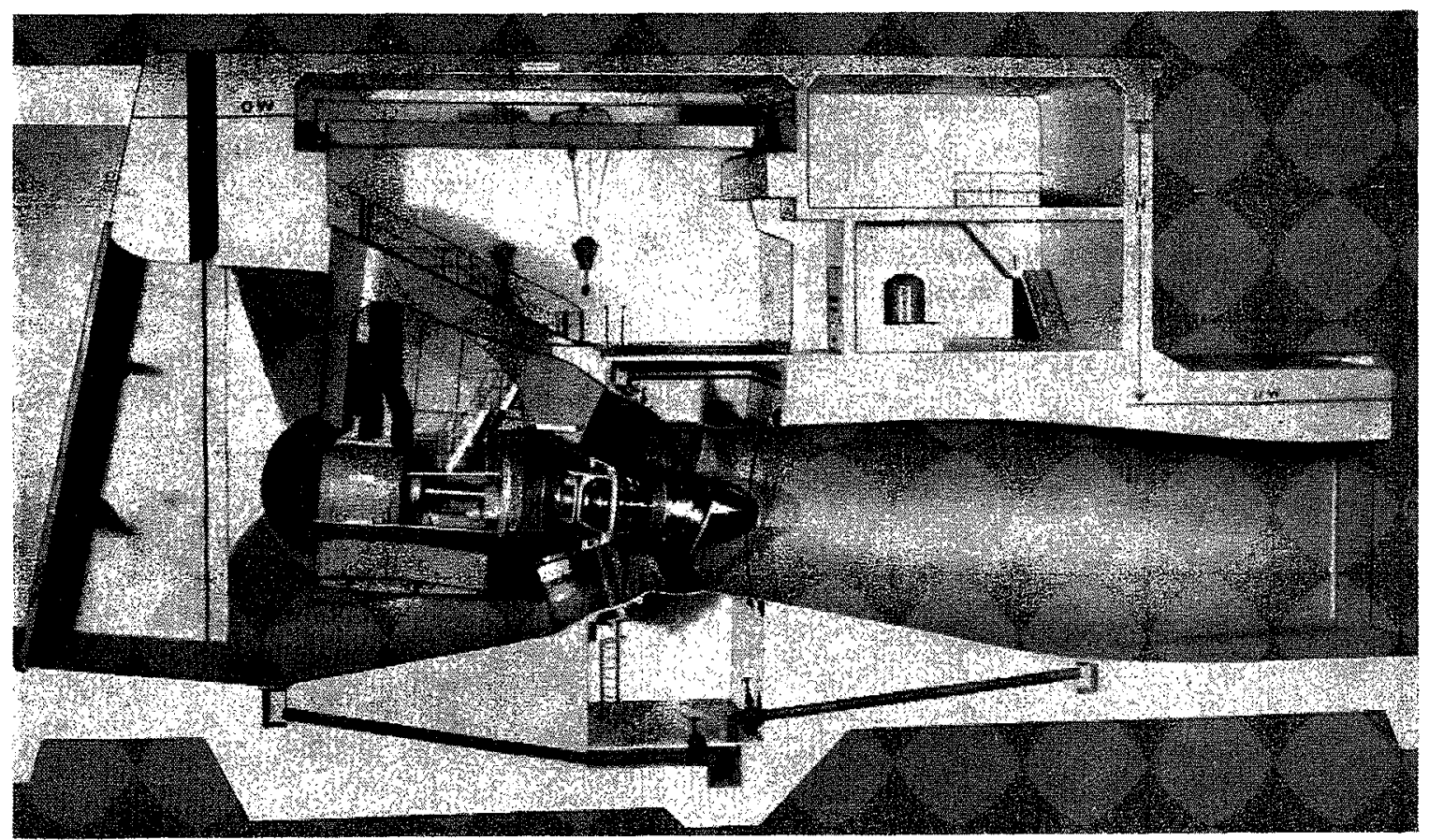

FIG. 5

Modèle d'une turbine axiale pour les centrales sur la Moselle. $\mathrm{Q}=380 \mathrm{~m}^{3} / \mathrm{s} ; \mathrm{H}=4,95 \mathrm{~m} ;$ puissance $=16 \mathrm{MW}$.

de $1200 \mathrm{~m}^{3} / \mathrm{s}$, les barrages doivent être ouverts afin d'éviter des dégâts causés par des remous dans l'étroite vallée de la Moselle.

La vallée de la Moselle étant encastrée très itroitement entre l'Eifel et le Hunsrück depuis 'Trèves jusqu'à son embouchure dans le Rhin, l'extension en largeur des installations s'en est trouvée limitée, situation qui est aggravée par la présence d'importantes routes et voies ferrées sur les deux rives de la Moselle.

Les dimensions des barrages sont imposées par les débits de crue à évacuer. Les largeurs des écluses sont déterminées par leur aménagement pour des bateaux de 1500 t. Il faut donc choisir des constructions ramassées.

La beauté du paysage de la vallée de la Moselle, encadrée par des coteaux complantés en vignes, imposait également de songer à la prolection des paysages et à se limiter dans l'établissement des projets. Des corrections de la Moselle, dont le cours décrit parfois des méandres, étaient inadmissibles. Il était donc indispensable d'adapter au paysage les barrages, les écluses et les centrales y compris les lignes de transport d'énergie. Le "type de construction pour la Moselle ", basé sur cette servitude, est caractérisé par la mise en œuvre de vannes-secteur immergées et de turbines axiales dans les centrales. Les vannes et piles du barrage seront submeryées lors des haules eaux. La tendance est en faveur de construclions assez plates et ramassées.

\section{4. - Adaptation au paysage.}

Plusieurs usines hydrauliques en Allemagne et dans d'autres pays européens sont construites de telle façon que le barrage et l'usine dépassent de beaucoup le niveau de la retenue. Ceci est le cas, par exemple, pour les centrales au fil de l'eau sur le Danube, le Main et le Weser. La photo $n^{\circ} 4$ montre que les installations de la centrale de Coblence, mise en service en 1951 , - plus particulièrement le portique de montage, - dépassent de beaucoup le plan d'eau. Cette forme de construction est imposée par les quatre turbines Kaplan prévues ici, avec un débit $Q=380 \mathrm{~m}^{3} / \mathrm{s}$ sous une chute $\mathrm{H}=4,2 \mathrm{~m}$.

La collaboration entre les ingénieurs chargés de l'établissement du projet, et les architectes, a permis de définir pour les autres aménagements prévus sur la Moselle des principes de réalisation qui s'adaptent harmonieusement au cadre de la Moselle et satisfont aux désirs légitimes du Comité de Protection du paysage. L'abaissement des superstructures a été obtenu, entre autres, par l'installation de groupes Kaplan à double réglage axés selon l'écoulement, et avec une légère inclinaison sur l'horizontale. Le bâti axial enfermant la turbine et le générateur repose sur un socle. La figure 5 montre un modèle de cette turbine axiale, mais avec un autre palier. I.es organes de la machine demeurent accessibles 
depuis les fosses de montage desservies par des galeries de service.

Le dessin de l'aménagement de Trèves, actuellement en construction, montre un exemple d'adaptation avantageuse au paysage, Il comporte quatre turbines axiales pour un dẻbit $\mathrm{Q}=380 \mathrm{~m}^{3} / \mathrm{s}, \mathrm{H}=4,95 \mathrm{~m}$, ce qui fournit une puissance de $16 \mathrm{MW}$ et une production annuelle de $80 \mathrm{GWh}$ environ (voir fig. 6).

\section{5. - Aménagement de Coblence.}

L'aménagement de la chute de Coblence était déjà commencé avant le début de la deuxième guerre mondiale. Après quelques modifications, il a été mis en service en 1951. La forme de construction adoptée à cette époque et comportant les quatre turbines verticales Kaplan déjà mentionnées, pour un débit $Q=380 \mathrm{~m} / \mathrm{s}$, $\mathrm{H}=4,2 \mathrm{~m}$, exigeait pour l'usine une longueur de $69 \mathrm{~m}$. Avec la nouvelle « forme de construction pour la Moselle », décrite plus haut, la longueur de l'usine serait réduite à $45 \mathrm{~m}$ avec l'installation de quatre turbines de même conception que les groupes verticaux de la chute de Trèves, ce qui représente une économie de $65 \%$ environ sur la longueur.

Mentionnons encore qu'en 1913 déjà une centrale au fil de l'eau a été construite sur la Moselle à $33 \mathrm{~km}$ en aval de Trèves, avec une accumula- tion journalièe el hebdomadaire, en complément d'une centrale fonctionnant à la honille, installée à Trèves en 1902 [21]. Cetle centrale (Dhronkraftwerk) a été munie en 1956 d'une pompe d'accumulation de 5 MW qui pompe de la Moselle pendant la nuit environ $4000 \mathrm{l} / \mathrm{s}$ dans un réservoir surélevé de $100 \mathrm{~m}$. La puissance totale de cette centrale est de $8 \mathrm{MW}$.

Une ancienne centrale se trouve également sur la Sarre près de Sarrebourg, utilisant une chute de $20 \mathrm{~m}$ de la Leuk, affluent de la Sarre.

Sur la Kyll - près de l'embouchure de la Moselle - les Services Municipaux de la ville de Trèves ont également fait construire une centrale au fil de l'eau comme centrale à charge constante et comme complément de l'usine thermique de Trèves en 1926. Aujourd'hui, évidemment, cette centrale nous parait petite. Avec un débit maximum de $16,8 \mathrm{~m}^{3} / \mathrm{s}$ et une chute utile de $3,47 \mathrm{~m}$, on a pu obtenir une puissance de $380 \mathrm{~kW}$ et une production annuelle de $1,7.10^{6}$ $\mathrm{kWh}$. Cette centrale sur la Kyll est toujours en service [21].

\section{6. - Situation et implantation de la chute de Trèves.}

Ia faible hauteur de passage du vieux pont romain de Trèves, qui ne permet pas de retenue, a déterminé la position de la chute de Trèves;

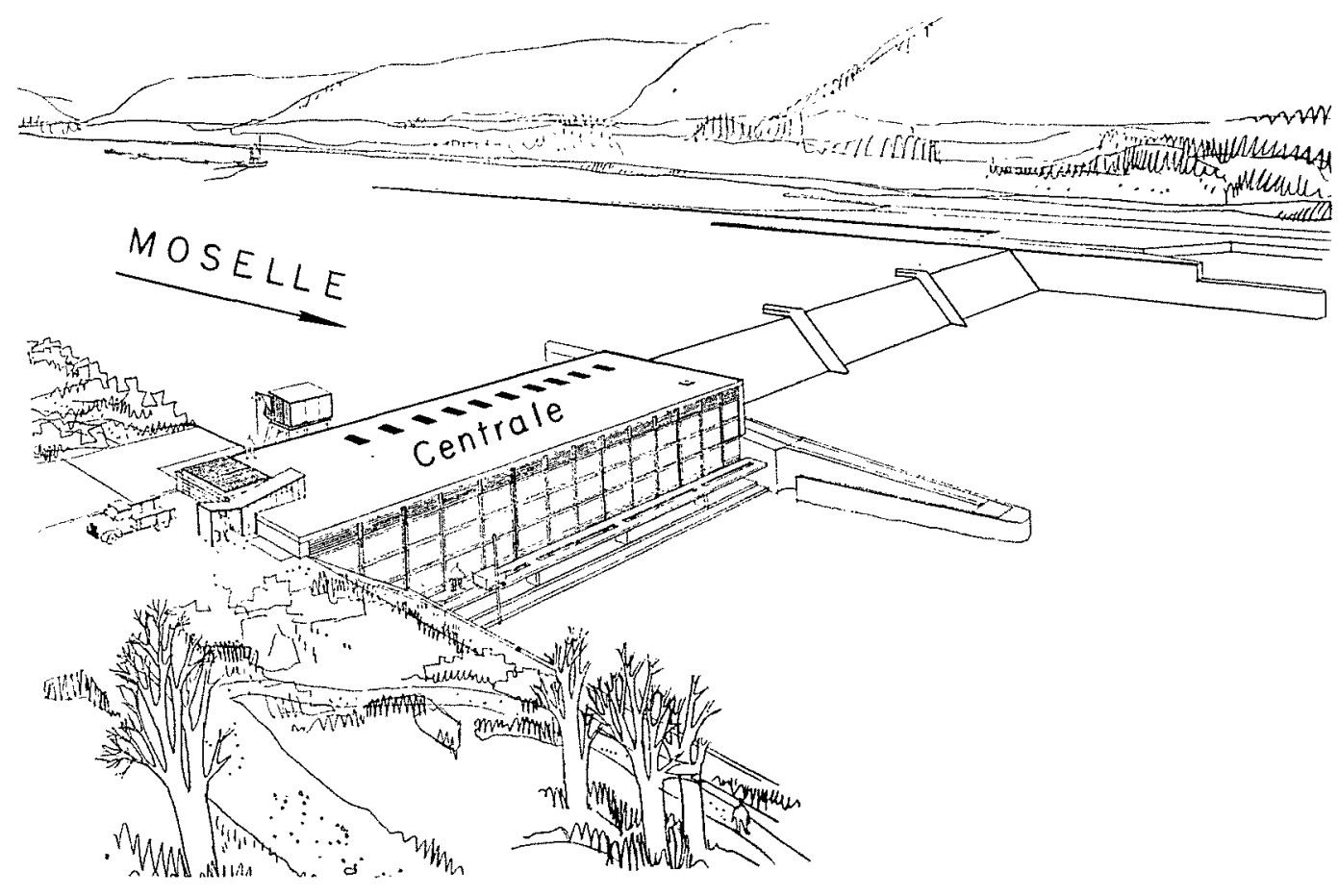

Frg. 6

vue de lamenagement de Treves avee sa centrale. 


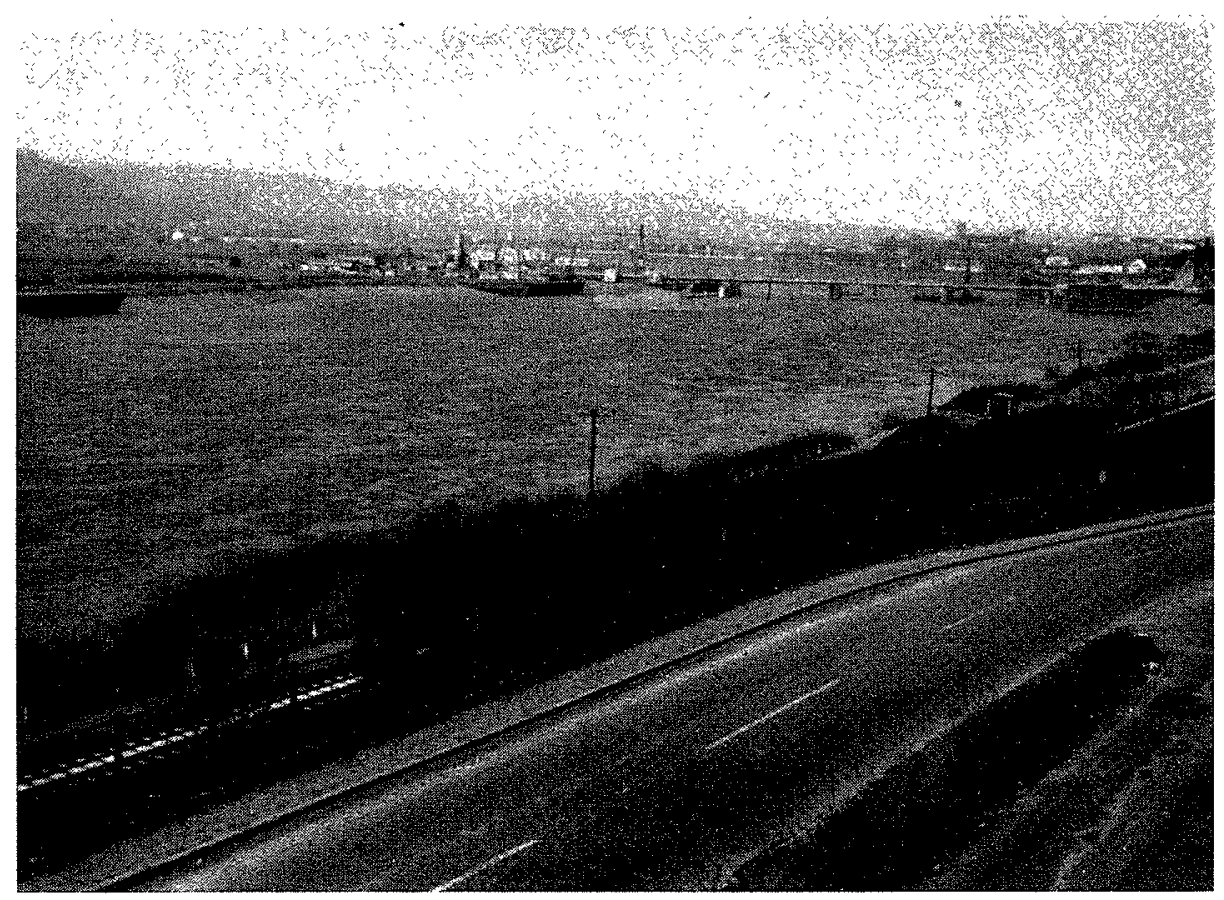

Fig. $\left.7 a_{1}\right)$

Vue générale près de l'amont.

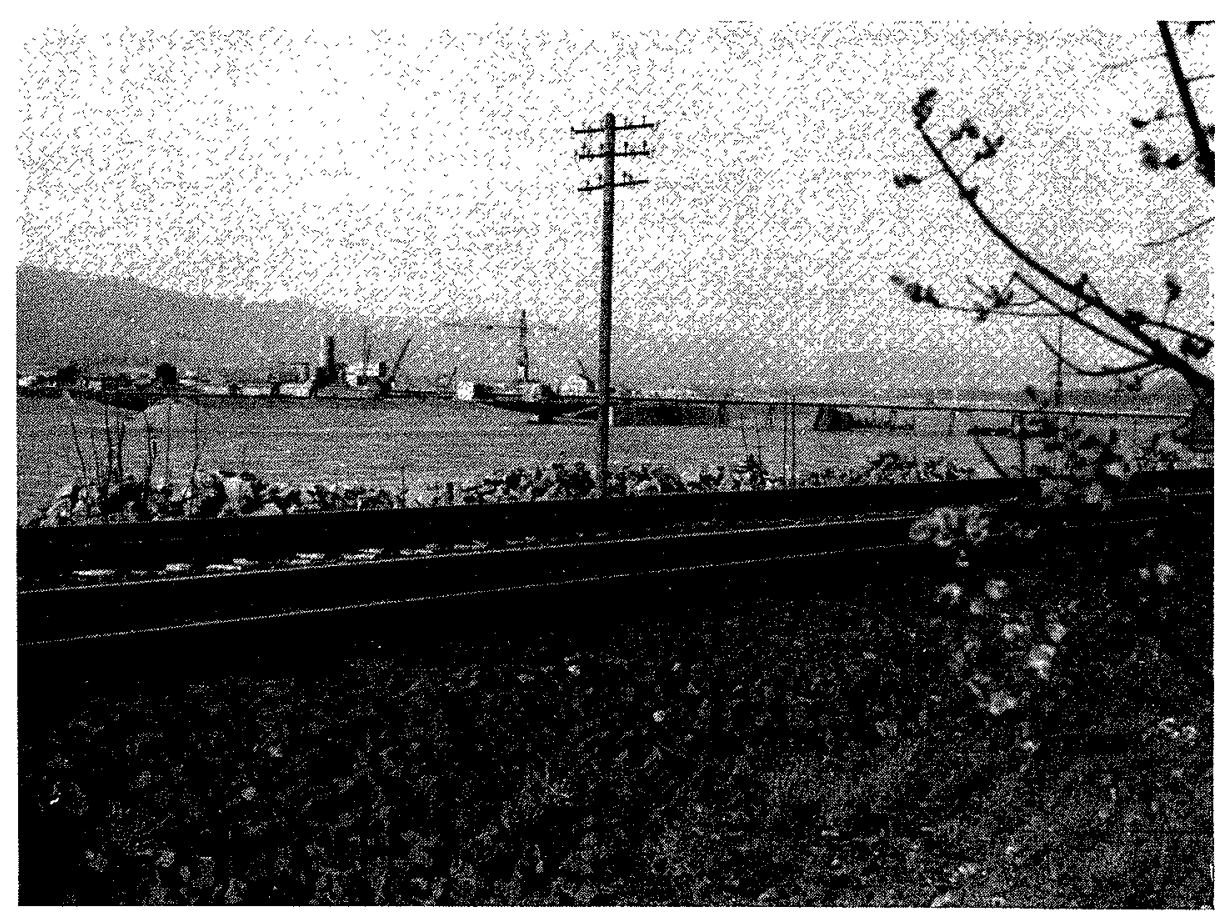

FIG $\left.7 a_{2}\right)$

Etage de retenue de Trèves en construction (état d'avancement en mars 1960). 


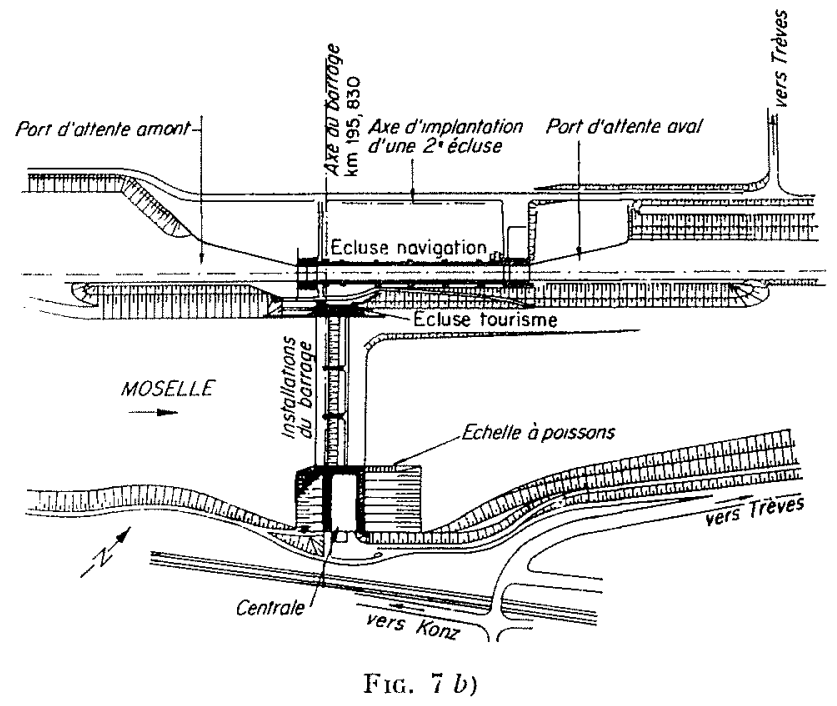

Plan d'enscmble de l'étage de retenue de Treves.

celle-ci fut implantée sur la Moselle en amont de ce pont au $\mathrm{km}$ 195.83. Le largeur de la rivière était ici suffisante et permettait une bonne adaptation des ouvrages au paysage [20].

Compte tenu de l'agglomération très étendue siLuée en amont, la hauteur de retenue a été fixée à $7,25 \mathrm{~m}$. L'asséchement de quelques parties de l'agglomération situées en contre-bas exige partiellement l'aménagement de surélévations et de digues ainsi que de réseaux de drainage et d'ouvrages d'épuisement.

La retenue s'étend jusque dans la Sarre qui se jette dans la Moselle près de Konz ainsi que jusque dans la Sure, dont l'embouchure est près de Wasserbillig. Sur l'Our - un affluent de la Sure --, le Benelux, la France, la Suisse ainsi que l'Allemagne de l'Ouest construisent à Vianden (Luxembourg) la plus grande centrale d'accumulation par pompage du monde, Le bassin amont, situé sur le Nikolausberg à $300 \mathrm{~m}$ au-dessus de la vallée de l'Our, aura une capacité d'accumulation de $3.10^{6} \mathrm{~m}^{3}$ d'eau. L'eau accumulée est amenée à la centrale souterraine par une conduite forcée d'un diamètre de $6,2 \mathrm{~m}$. La longueur totale de cette centrale est de $175 \mathrm{~m}$. Quatre unilés de $340 \mathrm{MW}$ et d'une production de pointe annuelle de $50010^{6} \mathrm{kWh}$ seront installées ici pendant la première période d'aménagement. La Société Electrique de l'Our S.A. (SEO), fondée pour l'aménagement, a limité le 15 juin 1959 son capital actions à $500000000 \mathrm{fr}$. avec une participation de $40 \%$ du Grand-Duché de Luxembourg. L'aménagement final prévoit pour cette installalion $800 \mathrm{MW}$. La mise en service de la première machine est prévue pour l'automne 1962 [22].

Le barrage de l'aménagement de Trèves s'étend sur toute la longueur de la rivière. L'écluse se trouve sur la rive gauche, en dehors du lit dans lequel la rivière coulait jusqu'à maintenant. La centrale est implantée dans la courbe de la rive droite. En même temps que cette inslallation, a été commencée celle de la chute de Delzem. La livraison des machines destinées aux aménagements de Trèves, Detzem el Lelımen a été áemandée. La photo 7 montre la disposition de la chute de Trèves.

\section{7. - Barrage de Trèves.}

Le barrage comporte trois pertuis, chacun ayant une largeur intérieure de $40 \mathrm{~m}$, et séparés par deux piles larges de 3,5 m [20]. L'écluse est reliée au barrage par une pile qui forme en mème temps une paroi de la chambre de l'écluse à péniches. La centrale est reliée au barrage par une pile comportant une échelle à poissons en forme d'escalier. La commande du barrage est entièrement hydraulique et il tourne autour d'un axe a charnières placé sur le côté aval.

Le corps de barrage fixe en béton est implanté profondément afin de réserver le logement de la vanne à secteur. Ce logement lui-même reçoil le corps de la vanne à secteur mobile en acier, lors. que des débits plus élevés exigent l'ouverture partielle ou complète des vannes [20] (voir photo 8 ).

Afin de pouvoir abaisser l'organe mobile de fermeture, le seuil du barrage a été muni d'une saillie d'une hauteur de $1,75 \mathrm{~m}$ au-dessus du lit de rivière et d'une forme favorable du point de vue hydraulique. Des essais sur modèle ont prouvé que ce rehaussement du seuil du barrage n'a pas une influence défavorable sur le transport des matériaux et l'évacuation des crues.

Il n'a pas été nécessaire de prévoir une passerelle au-dessus de l'eau, la galerie de service déjà mentionnće - dans le corps du barrage permettant la liaison entre l'écluse, le barrage el la centrale.

L'ouverture el la fermeture des vannes à secteur est obtenue par la pression même de l'eau en reliant les logements des secteurs au moyen de conduites dans les piles avec l'amont et l'aval.

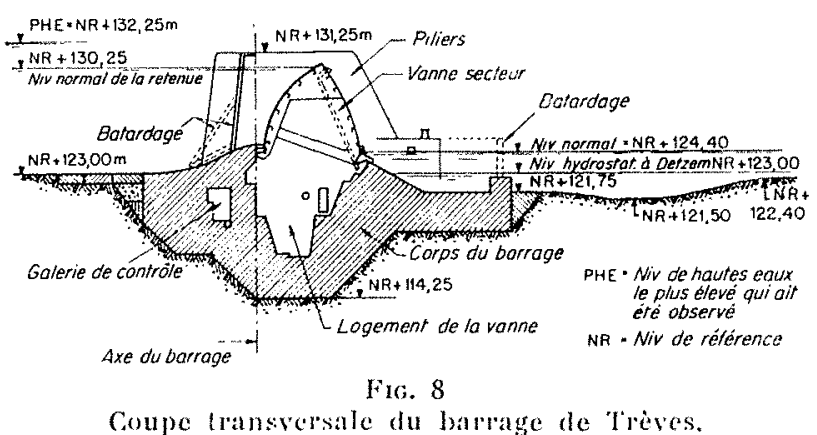


Les fermetures à secteur ne nécessitent donc pas de dispositifs de démarrage mécanique.

Une faible hauteur des piles, qui ne dépassent que de $1 \mathrm{~m}$ la cote de retenue normale en amont, est obtenue par ce système. Les piles sont accessibles par la galerie de service dans le corps du barrage.

Des tuyaux renfermant des fascines ont été ménagés dans les piles afin de permettre aux anguilles, qui ne remontent pas par l'échelle à poissons, de passer d'aval en amont.

Les piles du barrage, ainsi que toute l'installation d'écluse, sont submergés de $1 \mathrm{~m}$ lors des plus hautes eaux.

\section{8. - Installation d'écluses.}

L'installation d'écluses comporte l'écluse destinée au passage des grands bateaux de $1500 \mathrm{t}$ avec une longueur utile de $170 \mathrm{~m}$ et une largeur de $12 \mathrm{~m}$. D'autre part, elle comprend des ports d'attente et des jetées de séparation. L'écluse à péniches destinée aux petits bateaux a une longueur utile de $18 \mathrm{~m}$ et une largeur de $3,5 \mathrm{~m}$. Une seconde écluse à bateaux a été prévue du côté de la berge avec une longueur utile de $170 \mathrm{~m}$ et une largeur de $20 \mathrm{~m}$. Le port d'attente amont de la chute de Trèves possède déjà la largeur requise.

Afin de satisfaire aux exigences de la propulsion par pousseurs, qui nécessite des entrées et sorties rectilignes, les jetées de séparation forment un prolongement rectiligne de la paroi de l'écluse située du côté de la rivière.

Les dimensions de l'écluse à bateaux permettent le passage de deux bateaux de $1500 \mathrm{t}$ ayant chacun une longueur de $80 \mathrm{~m}$, une largeur de $10,5 \mathrm{~m}$ et un tirant d'eau de $2,5 \mathrm{~m}$ [20].

Le temps de remplissage ou de vidange de l'écluse est d'environ sept minutes avec un niveau aval moyen.

L'écluse à péniches se trouve directement à côté du barrage. L'entrée et la sortie en sont complètement séparées des installations destinées au passage des grands bateaux, ce qui évite que les embarcations ne se gênent mutuellement dans leurs manœuvres. Les portes busquées de l'écluse sont à un battant et à fonctionnement hydraulique. Leur fonctionnement est commandé directement par l'utilisateur.

Pour le passage des petits canots de sport, noLamment des nombreux berthons qui circulent pendant la saison d'été à travers la vallée pleine de charme de la Moselle, un passage a été prévu oì les sportifs peuvent porter leurs canots.

Il faut encore mentionner que l'écluse destinée aux grands bateaux sera de construction massive.

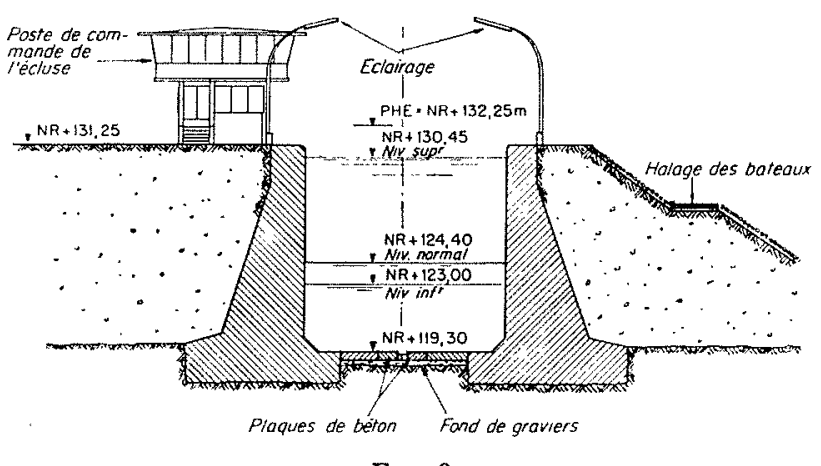

FIC. 9

Coupe transversale de l'écluse à chalands, largeur $12 \mathrm{~m}$, longueur utile $170 \mathrm{~m}$, pour des sateaux de $1500 \mathrm{t}$.

Les bajoyers sont ancrés par leur propre poids. Le fond perméable est simplement recouvert de plaques en ciment. La photo $\mathrm{n}^{\circ} 9$ montre la coupe transversale de l'écluse à bateaux. La tête amont est munie d'une porte qui peut être abaissée ou relevée et qui permet l'évacuation des crues. La tête aval est munie d'une porte busquée à fonctionnement hydraulique. Ceci supprime les postes de commande de chaque côté et, par suite, des constructions gênantes [20].

\section{9. - Centrale.}

Ainsi que nous l'avons déjà dit, la centrale est, comme le barrage et les installations d'écluse, de construction aussi basse que possible.

Les quatre turbines axiales, avec une capacité d'absorption $Q=380 \mathrm{~m}^{3} / \mathrm{s}$, ont déjà été menLionnées (voir photo $n^{\circ} 5$ ). Ces unités Kaplan à double réglage sont munies d'une transmission intermédiaire entre la turbine et la génératrice. Cette dernière se trouve dans un carter situé entièrement dans le courant. Pour des raisons hydrauliques, ce carter doit avoir un faible diamètre. Ceci réduit les dimensions de la génératrice, en permettant une grande vitesse de rotation. En effet, les hauteurs de chute d'eau sur la Moselle étant faibles, les vitesses de rotation des turbines sont de l'ordre de 70 à $90 \mathrm{tr} / \mathrm{mn}$. Pour ces motifs, des engrenages épicycloïdaux, donnant un rapport de 1 à 10 , ont été prévus. On obtient ainsi une faible perte de rendement, de 1 à $1,5 \%$ environ, qui est compensée par une vitesse de rotation plus élevée de la génératrice.

La puissance électrique maximale produite par les dix centrales, soit au total $165 \mathrm{MW}$, est reprise par le réseau interconnecté à haute tension fonctionnant en parallèle avec la France, le Benelux et les nations alpines. Cette puis sance totale peut se réduire à $10 \mathrm{MW}$ lors des basses eaux. Ia compensation est assurée au moyen du fonctionne- 


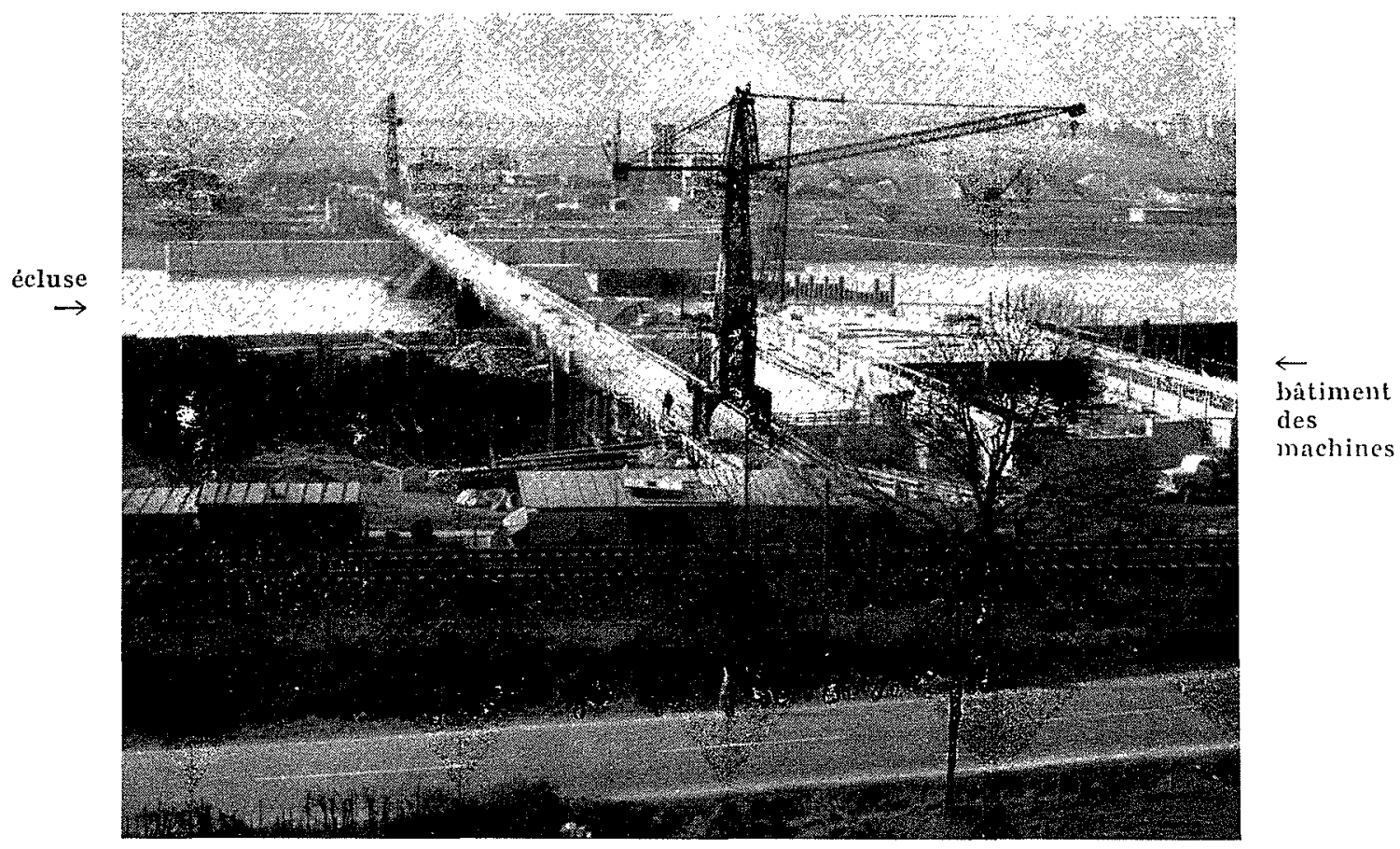

FIG. 10

Etage de retenue de Tréves en construction (état d'avancement en mars 1960).

ment par «éclusées » (voir les explications données plus loin).

\section{0. - Travaux de construction et frais.}

Les débits de la Moselle étant très élevés pendant les mois d'hiver, il n'est pas possible d'effectuer des travaux dans le lit de la rivière pendant cette période. Durant les autres saisons, le batardage pour un pertuis cause déjà un rétrécissement considérable de la section d'écoulement, ne permettant pas d'autres aménagements. Il en résulte, pour les trois pertuis, une durée de construction totale s'étendant sur trois étés, donc deux ans et demi au moins [20].

La construction de l'écluse et de la centrale qui, pour la majeure partie, se trouvent en dehors du lit de la rivière, peut s'effectuer aussi bien pendant les mois d'hiver.

Les travaux de construction pour le pertuis de gauche ont commencé au cours du mois de mars 1958. La construction des batardeaux ainsi que les travaux d'excavation ont été effectués pendant cette même période. D'autre part, on a procédé au hétonnage des seuils de barrage et des piles. Le montage des volets secteurs a été accéléré de façon à pouvoir procéder aux essais et à la mise en service des pertuis en novembre 1958 avant les crues hivernales. Pendant cette période, les tra- vaux de construction de l'écluse avaient été commencés et conduits de façon accélérée [20].

Le montage des portes d'écluse a eu lieu pendant l'été 1959. Comme le montre la photo 10, la construction de la centrale a commencé. Elle sera terminée pendant l'automne 1960 en même temps que celle du dernier pertuis.

Les délais de construction mentionnés sont extrêmement courts. L'aménagement du Main dure déjà depuis plus de cinquante ans. La canalisation du Neckar a été commencée en 1920. Quarante ans plus tard, le port de Stuttgart a été mis en service. Ces exemples montrent suffisamment la durée de construction remarquablement courte que l'on essaie d'obtenir pour la canalisation de la Moselle afin d'établir le plus vite possible la liaison entre la Lorraine et le bassin de la Ruhr ainsi que les ports maritimes.

Dans six à sept ans, la Moselle sera canaliséc sur une longueur de $270 \mathrm{~km}$ allant de Thionville à Coblence.

Ces importantes constructions - pour apporter ici quelques chiffres - comporteront, uniquement pour le barrage et l'écluse, sans tenir compte de la centrale :

- environ $500000 \mathrm{~m}^{3}$ de terrassements (terre et rocher);

- environ $65000 \mathrm{~m}^{3}$ de béton armé; 
- environ $30000 \mathrm{~m}^{*}$ de palplanches en acier;

- environ $650 \mathrm{t}$ d'ouvrages de montage en acier.

Les frais de construction de la chute de Trèves s'élèvent à 20 millions de DM environ. La totalité des frais pour les installations de navigation, les digues et les centrales s'élèveront - suivant le barème de construction actuel - à environ 650 millions de DM. Un peu plus de $40 \%$ de cette somme seront supportés par les sociétés francaises, environ $20 \%$ par l'Allemagne de l'Ouest.

\section{1. - Fonctionnement par éclusées.}

Nous avons déjà parlé des variations de débits de la Moselle (voir photo 3). Elles correspondent aux caractéristiques hydrologiques d'une rivière de montagne moyenne. Les variations vont de $25 \mathrm{~m}^{3} / \mathrm{s}$ au minimum à $4100 \mathrm{~m}^{3} /$ maximum.

Pendant les mois d'été, il existe de longues périodes de basses eaux avec des débits allant de $1 / 6^{\mathrm{e}}$ à $1 / 10^{\circ}$ du débit moyen $\left(25\right.$ à $\left.50 \mathrm{~m}^{3} / \mathrm{s}\right)$.

Le fonctionnement par éclusées - appliqué en Allemagne sur l'Isar, la Lech et le Neckar pallie cette réduction d'énergie : lorsque les débits sont faibles, l'eau est retenue dans le bassin amont et évacuée par plusieurs centrales au moment des besoins de pointe du réseau. Un bassin aval fait la compensation par l'accumulation de l'eau et son évacuation régulière vers l'aval. Le fonctionnement par éclusées s'étendra - après achivement des travaux d'installation - sur un troncon de la Moselle long de $200 \mathrm{~km}$.

Les possibilités d'accumulation $d u$ bassin amont près de Trèves et du bassin aval près de Coblence ne sont que de $1,6.10^{6} \mathrm{~m}^{3}$ pour chaque bassin. Compte tenu de la navigation, des variations du niveau de retenue supérieures à $0,6 \mathrm{~m}$ sont inadmissibles.

La puissance maximale de $165 \mathrm{MW}$ produite dans les dix centrales implantées sur la Moselle peut s'abaisser à $10 \mathrm{MW}$ lors des basses eaux. Le fonctionnement par éclusées permet une augmentation considérable (jusqu'à $110 \mathrm{MW}$ environ) dès possibilités de pointe, même lors des basses eaux. Une réduction de la hauteur de chute entraîne évidemment une réduction du nombre de kWh exploitables. Le fonctionnement par éclusées diminuera probablement de 4 à $6 \%$ environ la production moyenne d'énergie électrique annuelle. Les variations du débit seront cependant équilibrées par le système d'interconnexion nécessitant surtout des $\mathrm{kW}$ et non des $\mathrm{kWh}$ pour couvrir les besoins de pointe. Une amélioration des conditions peut être obtenue au moyen de l'accumulation par pompage.

\section{2. - Centrales d'accumulation par pompage.}

Nous avons déjà mentionné la petite centrale d'accumulation par pompage sur la Dhron, d'une puissance de $8 \mathrm{MW}$, ainsi que l'installation de Vianden, au Luxembourg, en cours de construction, qui aura, une fois terminée, une puissance totale de $800 \mathrm{MW}$.

Après l'achèvement des travaux de canalisation, d'autres centrales d'accumulation par pompage sont prévues sur la Moselle. La chute de 280 à $350 \mathrm{~m}$ due aux différents niveaux de la Moselle fournit des conditions naturelles favorables pour l'installation de telles centrales de pompage. Il faut encore ajouter que les collines étant parfois proches du lit de la Moselle, il s'ensuit de plus courtes liaisons entre le bassin amont et le bassin aval. D'autre part, les bassins de retenue du troncon de canalisation peuvent être utilisés en complément pour l'utilisation par pompage.

En vue de protéger le paysage, les futures centrales de pompage sur là Moselle seront aménagées dans le rocher (usines souterraines).

L'existence des diverses retenues, permettent l'aménagement de plusieurs centrales d'accumulation par pompage à grande puissance, dans la section située entre Trèves et Coblence. La centrale de pompage «Untermosel », entre MüdenLehmen-Coblence, est prévue pour quatre groupes de machines d'une puissance de 90 MW chacun. On dispose d'une chute utile moyenne de $300 \mathrm{~m}$ pour une puissance totale de $360 \mathrm{MW}$. Le débit maximal turbiné est de $150 \mathrm{~m}^{3} / \mathrm{s}$, le débit pompé de $80 \mathrm{~m}^{3} / \mathrm{s}$.

\section{BIBLIOGRAPHIE}

[1] Mathand. - Die Rheinlande, Bd. 2 : Die Mosel.

[2] Trinius (A.). -- Die Mosel und ihre Burgen (1926).

[3] Kretzschman (B.). - - Die Zukunft der europäischen Energiewirtschaft. Rheinischer Merkur, Koblenz, Nr. 39, v. 18-10-1947.
[4] Kretzschmar (B.). - Die Mosel im Rahmen evropäischer Planungsarbeiten. Trierische Volkszeituny, $\mathrm{Nr} .88$ (1948).

[5] KRETzSchiar (B.). - Mehr Elektrizitäts für Europa. Verstärkter Einsatz der Wasserkräfte zur Stromgewinnung. Allgemeine Zeitung Mainz, 19-9-1949. 
[6] Kretzchmar (B.). - Strommangel, eine europäische Erscheinung. Langfristige, umfangreiche Ausbaupläne in fast allen europäischen Ländern. Allgemeine Kölnische Zeitung, 8-10-1948.

[7] Kretzschmar (B.). - Ausbau von Wasserkräften zum Wiederaufbau Europas. Frankfurter Neue Presse, Nr. 165, v. 22-10-1948

[8] Kretzschmar (B.). - Strom zwischen Saar und Ruhr. Auftakt zur europäischen Verbundwirtschaft. Allgemeine Zeitung Mainz, 16-1-1948.

[9] Kretzschmar (B.). - Europa muss noch mehr Strom erzeugen. Stromeinschränkungen sind nicht nur eine deutsche Krise. Allgemeine Zeitung Mainz, Nr. 204, v. 4-11-1948.

[10] Kretzschmar (B.). - Stromerzeugung finanziert neue Wasserwege. Mosel und Rhein-Main-Donau Kanal werden ausgebaut. Allgemeine Zeitung Mainz, Nr. 244, v. 22-12-1948.

[11] Kretzschmar (B.). - Europäische Verbundwirtschaft Wirtschaftszeitung, Nr. 39, v. 24-9-1948.

[12] Kretzchmar (B.). - Europa muss noch mehr Strom erzeugen. Zeitung «Der Schlüssel», v. 14-8-1949.

[13] KRETzSCHMAR (B.). -- Was ist Verbundwirtschaft? Rheinischer Merkur, Koblenz, Nr. 8, v. 19-2-1949.
[14] Kretzschman (B.). - Die Bedeutung der primären Energieträger für die Struktur der öffentlichen Elektrizitätswirtschaft in Frankreich unter besonderer Berücksichtigung der technischen Probleme des Verbundbetriebes. Wiss. Techn. Hochschule Aachen, 1942.

[15] Energie Heute. Veröffentlichungen anlässlich der 5. Volltagung der Weltkraftkonferenz in Wien, 1956.

[16] Valerio (G.). - Prospettive -di sviluppo dell'industria elettrica in Europa. Quad. Studi el Notizie, Bd. 15 (1959), S. 503-511.

[17] Valerio (G.). - Perspectives de développement de l'industrie électrique en Europe. L'Economie électrique, vol. 33 (1959), $\mathrm{n}^{\circ} 20$, p. 119-133.

[18] Kretzschmar (B.). - Die Elektrizitätswirtschaft in den Vereinigten Staaten von Amerika bis 1975. VDI Nachrichten, Nr. 19, v. 12-9-1959.

[19] Pristerer (E.). - Erschliessung der Wasserkräfte in «Energie aujourd'hui ». 5. Volltagung der Weltkraftkonferenz, 1956, Wien, Bd. 2.

[20] Hormman (W.). - Vom Bau der Staustufe Trier. VDI Nachrichten, Nr. 8 v. 11-1-1959, Seite 9.

[21] Die Wasserkraftwirtschaft Deutschlands, Festschrift zur Weltkraftkonferenz, Berliu, 1930.

[22] Kretzschmak (B.). - Pumpspeicherwerk Vianden an der Our. BWK, 11 (1959), Nr. 12.

\section{Important bureau d'études recherche}

$1^{\circ}$ DESSINATEUR-PROJETEUR

spécialiste eau et assainissement. Résidence Paris

$2^{\circ}$ TOPOGRAPHE

ayant expérience observations hydrologiques

Résidence Outre-Mer

$3^{\circ}$ INGÉNIEUR V.R.D.

Résidence Outre-Mer

B.C.E.O.M., 90, boulevard Latour-Maubourg, PARIS

Important Bureau d'études recherche

INGENIEURS HYDRAULICIENS

spécialistes adduction d'eau

INGENIEURS E.T.P.

Ouvrage d'art béton armé pour études outre-mer

B.C.E.O.M., 90, boulevard Latour-Maubourg, PARIS 